\title{
Intersphincteric Resection for Low Rectal Cancer - Case Report
}

\author{
Russu Cristian¹, Molnar Călin1, Copotoiu Sanda Maria², Sărăcuț Claudiu1, Gherghinescu Mircea1, \\ Molnar Varlam Claudiư ${ }^{3}$, Copotoiu Constantin ${ }^{1}$

\begin{abstract}
1 Surgical Clinic 1 of the Emergency County Hospital and the University of Medicine and Pharmacy of Tîrgu Mures, Romania
${ }^{2}$ Anesthesia and Intensive Care Clinic 1 of the Emergency County Hospital and the University of Medicine and Pharmacy of Tîrgu Mureș, Romania
\end{abstract} \\ ${ }^{3}$ Obstetric-Gynecology Clinic, Clinical Emergency County Hospital Tirgu Mures, University of Medicine and Pharmacy Tirgu Mures, Romania
}

\begin{abstract}
Introduction: Surgical treatment for low rectal cancer represents a challenge: to perform a radical resection and to preserve the sphincter's function. We report a case of intersphincteric resection in a combined multimodality treatment for low rectal cancer, with good oncologic and functional outcome. Case presentation: We report a case of a 73 years old woman admitted in April 2014 in surgery, for low rectal cancer. The diagnostic was established by colonoscopy and malignancy confirmed by biopsy. Complete imaging was done using computed tomography and magnetic resonance to establish the exact stage of the disease. The interdisciplinary individualized treatment began with radiotherapy (total dose of 50 Gy, administered in 25 fractions) followed by surgery after eight weeks. We performed intersphincteric rectal resection by a modified Schiessel technique. There were no postoperative complications and the oncologic and functional results were very good at one year follow up. Conclusions: Intersphincteric resection, in this selected case of low rectal cancer, represented an efficient surgical treatment, with good functional results and quality of life for the patient. A multidisciplinary team is an invaluable means of assessing and further managing the appropriate, tailored to the case, treatment in the aim of achieving best results.
\end{abstract}

Keywords: intersphincteric resection, low rectal cancer, interdisciplinary, radiotherapy

Received: 23 August 2015 / Accepted: 30 October 2015

\section{Introduction}

There have been great advances in understanding and treatment of rectal cancer in the last few decades $[1,2]$. Nowadays the management of rectal cancer recognizes a multimodality approach $[3,4]$. With all the major progresses made in the development of oncologic therapy, the radical surgical removal of the tumor stays, however, the only treatment in order to achieve a permanent cure $[3,5]$. Besides the oncologic resection of the tumor, the second most important goal is the preservation of fecal continence [5].

The surgery for rectal cancer focuses today on functional preservation of the anal sphincter [2,3]. This concept was embedded paralleling to the discovery that the cephalic lymphatic stream was the main way of dissemination in rectal cancers $[5,6]$. Both the Heald's concept of total mesorectal excision and the use of preoperative radiotherapy, evidenced to decrease of the local recurrence rate, encouraging furthermore the development of sphincter saving procedures $[1,3,5,6]$.

If for proximal rectal tumors the surgery is considered to be easier, low rectal tumors represent a challenge even for highly experienced colorectal surgeons [7].

The intersphincteric resection technique used to increase sphincter preservation by achieving the necessary distal margin, is regarded as the most extreme form of resection for "ultralow" rectal cancers [5,7].

${ }^{*}$ Correspondence to: Călin Molna

E-mail: molnar.calin@yahoo.com
We report a case of low rectal cancer managed by multimodal treatment. The surgery consisted in intersphincteric resection followed by good functional and oncologic outcome.

\section{Case report}

We report a case of a female patient aged 73 years admitted in the Surgical Clinic 1 of the County Emergency Clinical Hospital of Tîrgu Mureș, in April 2014, with the diagnostic of low rectal cancer.

The onset of the disease was four months before the admission, with the emergence of mild lower abdominal pain, accompanied by changes of intestinal transit and rectal hemorrhage episodes.

Due to recurrence of rectal bleeding episodes, she was submitted to a surgical examination, which, after performing a digital rectal examination, raised the suspicion of a rectal tumor located at approximately $6 \mathrm{~cm}$ from the anal orifice. A colonoscopy was consecutively recommended.

The colonoscopic examination detected a sessile polypoid formation, centrally depressed, at about $6 \mathrm{~cm}$ from the anal orifice and a biopsy was taken for the suspicion of rectal cancer.

Three weeks later, the histopathological result stated the diagnosis of "well differentiated, infiltrative, rectal adenocarcinoma" and additional imaging examinations were recommended to establish the staging of the disease.

The computed tomography (thorax, abdomen and pelvis) with intravenous dye, detected no local and regional 
pathological lymph nodes, distant lung or liver metastases. A magnetic resonance imaging (MRI), of the pelvis described a lesion located in the left lateral wall of the lower rectum, relatively well defined, not exceeding the muscular layer, without extension to the perirectal adipose tissue and mesorectal fascia, without pathological pelvic lymph nodes.

At that point, the surgeon and the oncologist, following a joint analysis of the pooled data, staged the patient a well differentiated (GI) low rectal cancer in stage I (T2 N0 M0) - AJCC classification (American Joint Committee on Cancer), or B1, according to Dukes classification (modified by Astler and Coller). The first step in therapeutic management must be the oncologic treatment (radiotherapy) followed by surgery.

The patient underwent preoperative exclusive radiotherapy for five weeks. The total dose of $50 \mathrm{~Gy}$, administered in 25 fractions, was well tolerated.

Eight weeks after the end of radiotherapy, the patient was admitted in the Surgical Clinic 1 of the County Emergency Clinical Hospital of Tîrgu Mureș, for surgical treatment.

After a preoperative mechanical bowel preparation with Macrogol 4000 (Fortrans ${ }^{\circ}$ ), with the patient in a LloydDavies position, the procedure began with the abdominal time. An inferior median laparotomy that allowed for a general and local inspection confirmed the preoperative evaluation. We decided to proceed with surgery. Due to the low location of the tumor and the necessity of respecting the oncologic principles, we decided to perform an intersphincteric rectal resection using a modified Schiessel

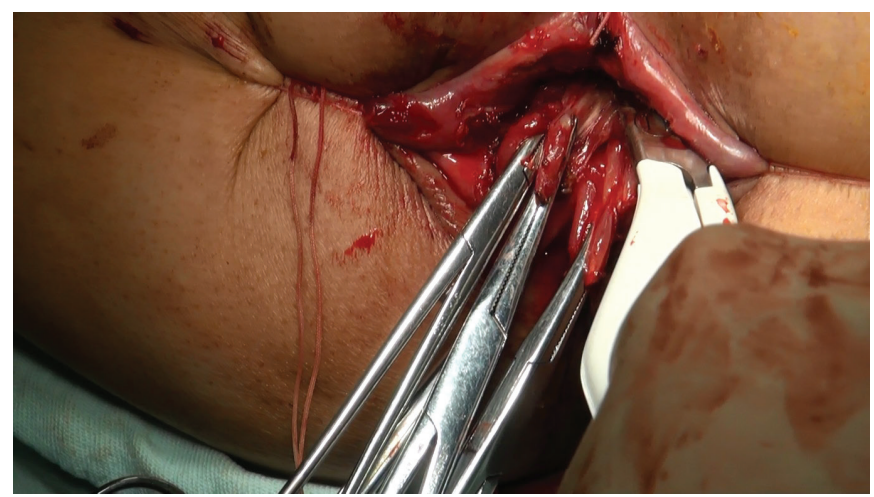

Fig. 1. Intersfincterian removal of the anal canal with LigaSure Small-Jaw

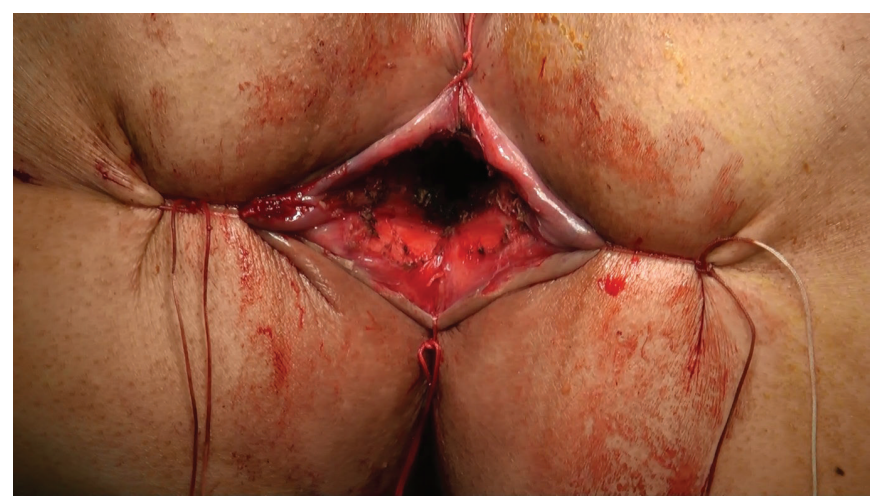

Fig. 3. Final aspect - the remaining external anal sphincter technique. We began with the high sealing and cutting of the inferior mesenteric vessels and left colon mobilization, including the splenic flexure, without ligatures. A total mesorectal excision was achieved, with sharp dissection using LigaSure Impact ${ }^{\mathrm{TM}}$, preserving the presacral autonomic nerves.

Next, the internal sphincter was incised and separated from the external sphincter and puborectalis muscle (Figure 1). A circular dissection of the rectum containing the tumor, following the intersphincteric anatomical plane, was then done, using a LigaSure Small Jaw device (Figure 2, Figure 3)

As the final time, by pulling through the descending colon, a hand sutured coloanal anastomosis was performed using absorbable sutures, placed in between the colon, the external sphincter, and the anoderm (Figure 4). This was the only operative time when we used threads.

The postoperative evolution was favorable, without any complications, and the patient was discharged eight days after surgery.

The histopathological result established the diagnostic of moderate differentiated (GII) rectal carcinoma, with mucinous content, infiltrating the rectal wall up to the muscular layer. All the resection margins (distal, proximal and circumferential) were found free of tumor. No pathological lymph nodes were found and no vascular, lymphatic or perineural invasion was observed.

According to this result, the oncologic reevaluation decided that no additional oncologic postoperative treatment was necessary.

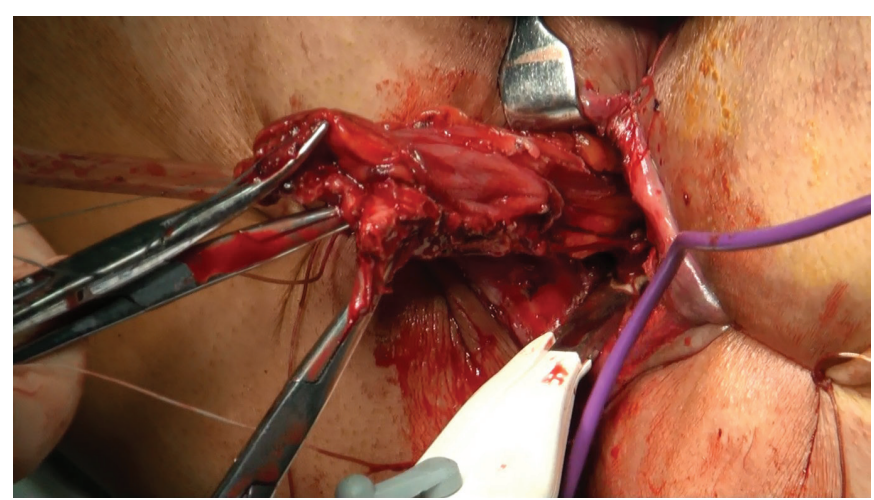

Fig. 2. Intersfincterian removal of the anal canal and inferior rectum with LigaSure Small-Jaw

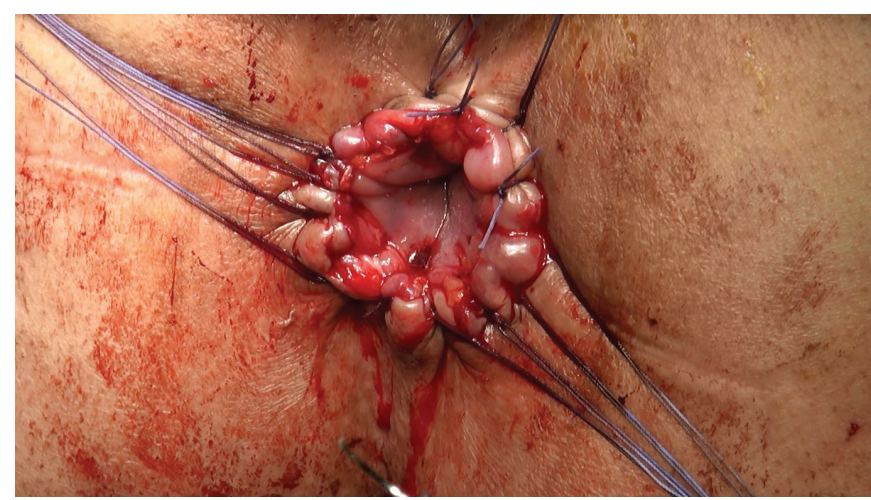

Fig. 4. Final aspect of the colo-cutaneous anastomosis 
The follow up of the patient was conducted both by the surgeon and the oncologist to establish the functional and oncologic result of the treatment. She was evaluated clinically, biologically and imagistically every three months up to a year.

No sign of local, regional or distal recurrence was detected in the ultrasound evaluation at three, six and nine months nor in the one year MRI. The levels of CEA (Carcinoembryonic antigen) were also within the normal range at each three month assessment.

The postoperative functional outcome evaluated according to the Waxner classification [8] registered a score of 6 points, the patient describing a good continence for solid stool, and intermittent incontinence for liquid stools and gases.

The patient was included, under a written informed consent, in a study about sphincter saving procedures performed for low rectal cancer, approved by the Hospital Ethics Committee.

\section{Discussions}

For this case of low rectal cancer, an intersphincteric resection was chosen as surgical procedure due to the low location of the tumor and the necessity of respecting oncological principles in trying to preserve the anal continence $[2,3,5]$.

All patients with indication of intersphincteric resection for low rectal cancer must be preoperatively evaluated clinically and imagistically to meet Schiessel's criteria: tumor located in lower rectum and anal canal, local spread restricted to the rectal wall or internal sphincter, histology G I or G II grading, absence of distant metastases and preserved sphincter function $[3,5]$.

Preoperative radiotherapy was performed to improve local control and the chance of performing a sphincter saving procedure $[1,9,10]$.

The association with preoperative chemotherapy is used to increase the efficacy of radiation, but produces no significant effect upon survival $[1,10]$. In our case no chemotherapy was associated due to a persistent leucopenia consistent throughout the entire therapy.

To respect the oncological principles of rectal surgery, a total mesorectal excision was performed and a free distal margin of at least $2 \mathrm{~cm}$ from the macroscopic limits of the tumor was achieved $[2,3]$.

The oncologic radicality of the technique was confirmed by the pathological report that showed a proximal, distal and also circumferential margin free of tumor.

In our procedure without sutures, the use of a LigaSure $^{\mathrm{TM}}$ device showed certain benefits in performing a good hemostasis and lymphostasis and allowing also a good control in performing the internal sphincter resection by approaching of the intersphincteric space in a correct, unmodified anatomic plane $[11,12]$.

We also considered that neither a protective stoma (ileostomy or colostomy) was necessary, as suggested by some authors $[9,13]$, because of the sub peritoneal location of the coloanal anastomosis.

From the functional point of view, the patient described a good continence for solid stools, and intermittent incontinence for liquid stools and gases. She achieved an overall state of good continence by using a diet designed to increase the consistency of the stools. When asked, she assessed the quality of her life at approximately $80-90 \%$ from that of the previous, free of disease state.

\section{Conclusions}

Intersphincteric resection in a selected case of low rectal cancer, represented an efficient surgical treatment, followed by both good functional results and a good quality of life for the patient.

The assessment of the case in a multidisciplinary team, the appropriate surgical and oncologic, tailored to the case, management were the most important factors in achieving a good oncologic and functional outcome.

\section{Acknowledgment}

This paper is supported by the Sectoral Operational Programme Human Resources Development (SOP HRD), financed from the European Social Fund and by the Romanian Government under the contract number POSDRU/159/1.5/S/133377/.

The study was conducted also under the framework of the research grant: "Sphincter saving procedures in female patients with low rectal cancer - a comparative study on the efficiency of LigaSure ${ }^{\mathrm{TM}}$ and mechanical sutures devices" (nr.:13136/23.09.2014), financed by: S.C. Three Pharm S.R.L. Tîrgu Mureș and developed by Tîrgu Mureș University of Medicine and Pharmacy.

\section{References}

1. Bisceglia G, Mastrodonato N, Tardio B, et al. Intermediate neoadjuvant adiotherapy for T3 low/middle rectal cancer: Postoperative outcomes of a non-controlled clinical trial. 4294367 Oncotarget. 2014;5(22):1114311153.PMCID:4294367

2. Mulsow J, Winter DC. Sphincter preservation for distal rectal cancer - a goal worth achieving at all costs? World J Gastroenterol 2011;17(7):855861.doi:10.3748/wjg.v17.i7.855

3. Spanos C P. Intersphincteric resection for low rectal cancer: An overview. Int J Surg Oncol. 2012;2012:241512.doi:10.1155/2012/241512

4. Musio D, De Felice F, Bulzonetti N, et al. Neoadjuvant-intensified treatment for rectal cancer: Time to change? World J Gastroenterol. 2013;19(20):3052-3061.doi:10.3748/wjg.v19.i20.3052

5. Schiessel R, Novi G, Holzer B, et al. Technique and long-term results of intersphincteric resection for low rectal cancer. Dis Colon Rectum 2005;48:1858-1867.doi:10.1007/s10350-005-0134-5

6. Heald R J. The 'holy plane' of rectal surgery. J R Soc Med 1988;81(9):503508.doi:10.1177/014107688808100904

7. Cipe G, Muslumanoglu M, Yardimci E, Memmi N, Aysan E. Intersphincteric resection and coloanal anastomosis in treatment of distal rectal cancer. Int J Surg Oncol. 2012;2012:581258.doi:10.1155/2012/581258

8. Jorge JM, Wexner SD. Etiology and management of fecal incontinence. Dis Colon Rectum. 1993;36(1):77-97

9. Deo S V S, Kapali A S, Gupta M, Shukla N K. A review of controversies in the management of colorectal cancers. Indian J Surg. 2012;74(3):221227.doi:10.1007/s12262-012-0586-5

10. Rullier E, Goffre B, Bonnel C, Zerbib F, Caudry M, Saric J. Preoperative radiochemotherapy and sphincter-saving resection for T3 carcinomas of the lower third of the rectum. Ann Surg. 2001;234(5):633-640. PMCID:1422087 
11. Araki Y, Noake T, Kanazawa M, et al. Clipless hand-assisted laparoscopic total colectomy using LigaSure AtlasTM. Kurume Med J. 2004;51(2):1058.doi.org/10.2739/kurumemedj.51.105

12. Manouras A, Filippakis G M, Tsekouras D, et al. Sutureless open low anterior resection with total mesorectal excision for rectal cancer with the use of the electrothermal bipolar vessel sealing system. Med Sci Monit,
2007;13(5):CR224-230,PMID:17476194

13. den Dulk M, Marijnen C A, Collette L, et al. Multicentre analysis of oncological and survival outcomes following anastomotic leakage after rectal cancer surgery. $\mathrm{Br} J$ Surg. 2009;96(9):1066-75.doi:10.1002/ bjs. 6694 